\title{
EXPERIÊNCIAS DE POPULARIZAÇÃO DE PLANTAS ALIMENTÍCIAS NÃO CONVENCIONAIS NO ESTADO DE ALAGOAS, BRASIL
}

\author{
POPULARIZATION EXPERIENCES OF UNCONVENTIONAL \\ FOOD PLANTS IN THE STATE OF ALAGOAS, BRAZIL \\ Gabriela Maria Cota dos SANTOS ${ }^{1}$, Déborah Monteiro BARBOSA ${ }^{1}$, Élida Monique da Costa SANTOS1, Danúbia Lins \\ GOMES $^{1}$, Rafael Ricardo Vasconcelos da SILVA ${ }^{1}$, Patrícia Muniz de MEDEIROS ${ }^{1 *}$ \\ ${ }^{1}$ Universidade Federal de Alagoas, Campus de Engenharias e de Ciências Agrárias, Laboratório de Ecologia, Conservação e Evolução Biocultural \\ *patricia.medeiros@ceca.ufal.br
}

\section{RESUMO}

Submitted: 22/07/2020; Accepted: 08/09/2020

Os seres humanos vêm restringindo a diversidade de alimentos em sua dieta ao longo dos anos, devido ao processo de globalização dos sistemas agroalimentares. Neste sentido, a promoção da popularização de plantas alimentícias não convencionais (PANC) pode ter importantes implicações nutricionais, ecológicas e socioeconômicas. Neste artigo buscamos descrever algumas estratégias de popularização das PANC no Estado de Alagoas realizadas pelo Laboratório de Ecologia, Conservação e Evolução Biocultural (LECEB) da Universidade Federal de Alagoas e como estas estratégias foram orientadas pelas pesquisas do grupo sobre o tema. Discutimos quatro grupos de estratégias, sendo estas: (1) apresentação aos potenciais consumidores de quatro PANC selecionadas a partir da percepção local de agricultores do Assentamento Dom Hélder (Murici-AL); (2) realização de minicursos com agricultores e extrativistas do assentamento Dom Hélder, do Bacharelado em Agroecologia (PRONERA) e com outros estudantes das ciências agrárias; (3) realização do concurso de culinária PANC na Pança; e (4) produção de vídeos de divulgação das PANC. Acreditamos que estas estratégias, somadas com outras iniciativas de pessoas e instituições diversas de Alagoas, têm ampliado o conhecimento sobre este grupo de espécies no Estado.

Palavras-chave: Avaliação sensorial, Diversificação alimentar, Etnobotânica, Conservação Biocultural

\begin{abstract}
Due to the globalization process of agrifood systems, human beings have been restricting food diversity of their diet over the years. Therein, the promotion of the popularization of unconventional food plants (UFP) can have important nutritional, ecological and socioeconomic implications. Thus, this article seeks to describe the UFP popularization strategies in the state of Alagoas that were carried out by the Laboratory of Ecology, Conservation and Biocultural Evolution (LECEB) of the Federal University of Alagoas and how the group's research on the subject guided these strategies. We discussed four groups of strategies: (1) the presentation to potential consumers of four UFP selected from the local perception of farmers from the Dom Hélder Settlement (Murici-AL); (2) holding minicourses with farmers and extractivists from the Dom Hélder settlement, from the Bachelor of Agroecology (PRONERA) and with other students of the agrarian sciences; (3) holding the cooking contest "PANC na Pança"; and (4) producing videos to promote UFP. We believe that these strategies, together with other initiatives by different people and institutions in Alagoas, have increased knowledge about this group of species in the state.
\end{abstract}

Keywords: Sensory evaluation, Food diversification, Ethnobotany, Biocultural conservation

\section{INTRODUÇÃO}

A humanidade vivencia um progressivo processo de homogeneização alimentar, havendo em sua dieta pouca diversidade de espécies. Estima-se, por exemplo, que três quartos da alimentação mundial sejam provenientes de apenas 12 espécies vegetais e 5 espécies animais (FAO, 2015). Esses valores são alarmantes, considerando que estimativas conservadoras apontam que pelo menos $10 \%$ das espécies vegetais conhecidas possui potencial alimentício (RAPOPORT et al., 1998). O Brasil, por sua 
vez, detém a maior biodiversidade do mundo com aproximadamente 15 a $20 \%$ das espécies do planeta (KINUPP e LORENZI, 2014), sendo um importante repositório da diversidade alimentar mundial.

Neste contexto, o conceito de plantas alimentícias não convencionais (PANC) vem ganhando popularidade no Brasil para referir-se a plantas de uso alimentício que não são comuns no dia-a-dia da grande maioria das pessoas de uma região, de um país, ou mesmo do mundo (KINUPP e LORENZI, 2014). Muitas PANC possuem quantidades expressivas de micronutrientes, que podem ser escassos nos cultivos de alta produção global (FAO, 2017). Além dos benefícios à saúde e dieta, as PANC de ocorrência natural estão livres do uso de agrotóxicos e fertilizantes, o que é especialmente importante em países que mantém um expressivo consumo destes insumos, como é o caso do Brasil (SOARES e PORTO, 2009).

Ainda, a popularização do consumo de plantas alimentícias não convencionais, além de contribuir para a consolidação de dietas diversificadas e menos dependentes da produção global, também se constitui em oportunidades para comunidades rurais. Para estas, o cultivo e extração de PANC podem fornecer um aporte de renda e diminuir a vulnerabilidade socioeconômica. Além disso, o contexto atual de incertezas climáticas e seu impacto na produção agrícola fortalecem ainda mais a necessidade de buscar produtos alternativos. Assim, as PANC poderiam conferir uma fonte adicional de renda em contextos de anos climaticamente atípicos, tendo em vista que muitas destas espécies ainda permanecem provendo os produtos alimentícios enquanto as produções agrícolas convencionais são perdidas. Exemplo disso é a palma forrageira (Opuntia ficus-indica Mill.), espécie altamente tolerante à seca e bastante disseminada na caprinovinocultura do semiárido brasileiro (MOREIRA et al., 2016), mas que vem sendo desaproveitada como alimento humano.

Outro ponto favorável à popularização das plantas alimentícias não convencionais é a sua adequação aos modelos de produção agroecológicos. Uma das ideias centrais da agricultura sustentável é espelhar-se, até onde possível, nos ecossistemas naturais, de modo a evitar remediações que incluam, por exemplo, o uso de insumos como agrotóxicos e fertilizantes (MAGDOFF e VAN ES, 2009). Nesse sentido, é recomendada a promoção de culturas agrícolas mais ajustadas ao contexto agroclimático e ambiental local. Muitas PANC são resistentes às pragas, seca e doenças, tornando-as particularmente convenientes para o cultivo orgânico (DUARTE, 2017; RANIERI et al., 2017).

Porém, especialmente em áreas urbanas, a popularização de alimentos não conhecidos pela maioria das pessoas, como é o caso das PANC, possui algumas barreiras. Desse modo, acreditamos que um bom caminho para o sucesso de programas de popularização de PANC é o de guiá-los por pesquisas científicas que identifiquem as principais barreiras e oportunidades para o consumo de PANC.

Assim, buscamos neste artigo apresentar algumas estratégias de popularização das PANC no Estado de Alagoas, que foram realizadas pelo Laboratório de Ecologia, Conservação e Evolução Biocultural (LECEB) da Universidade Federal de Alagoas e como estas estratégias foram orientadas pelas pesquisas do grupo sobre o tema. Apresentaremos quatro estratégias, sendo estas: (1) a degustação de PANC por frequentadores de feiras de Maceió (AL) e estudantes da Universidade Federal de Alagoas; (2) realização de minicursos com agricultores, extrativistas e estudantes de ensino superior; (3) realização do concurso de culinária PANC na Pança; e (4) produção de vídeos de divulgação das PANC.

\section{DEGUSTAÇÃO E AVALIAÇÃO SENSORIAL DE PANC}

As estratégias de popularização que relatamos no artigo fizeram parte do projeto Bases para o estabelecimento de um programa de popularização das plantas alimentícias não convencionais (CNPq proc. 442810/2016-4). O projeto caminhou por duas vertentes, sendo estas a disseminação de informações sobre PANC de uma maneira geral e a promoção de quatro espécies de PANC em particular. Um dos principais intuitos do projeto foi o de buscar uma ampliação no comércio de PANC pelos moradores do assentamento Dom Hélder Câmara (Murici-AL). Deste modo, as espécies foram selecionadas a partir de um protocolo de pesquisa que levou em conta o conhecimento e a percepção sobre as PANC pelos moradores. A partir deste protocolo, identificamos quatro espécies com maior potencial para popularização segundo indicativo local: Psidium guineense Sw. (araçá), Genipa americana L. (jenipapo), Xanthosoma sagittifolium (L.) (taioba) Schott e Dioscorea trifida L.f. (inhame da mata).

Realizamos degustações com 262 pessoas entre frequentadores de feiras agroecológicas e convencionais da região metropolitana de Maceió (AL) e estudantes do Campus de Engenharias e Ciências Agrárias da Universidade Federal de Alagoas. Estas degustações (Figura 1) serviram ao 
mesmo tempo para coletar dados de aceitabilidade e auxiliar na familiarização de potenciais consumidores com as PANC, apresentando diferentes formas de preparo. Apresentamos para os voluntários (um produto por voluntário) o suco de araçá, suco de jenipapo, pudim de inhame da mata e bolinho de taioba. As informações específicas sobre as PANC de cada receita só foram reveladas aos voluntários após a avaliação sensorial. Seguimos os métodos usados em estudos do tipo (LAWLESS e HEYMANN, 2010), de forma que os voluntários avaliaram a cor, textura, cheiro, sabor e avaliação geral do produto utilizando uma escala hedônica cujos extremos foram 1 ("desgostei extremamente") e 9 ("gostei extremamente").

A partir desta técnica observamos que, de um modo geral, as pessoas avaliaram bem os produtos a base de PANC, com todas as notas acima de sete (médias para avaliação geral de 8,52 para o araçá, 8,17 para o jenipapo, 7,95 para o inhame da mata e 7,41 para a taioba), sendo estes valores muitas vezes superiores a produtos com plantas convencionais (CARVALHO et al., 2005; VASCONCELOS et al., 2006; BERILLI et al., 2011).

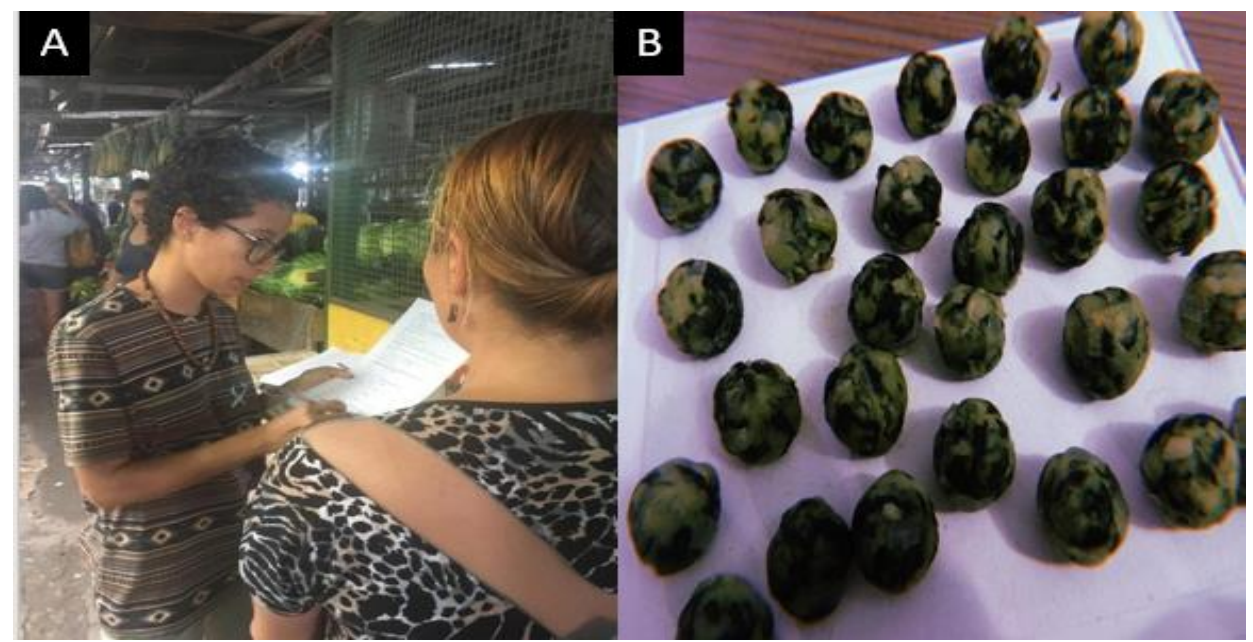

Figura 1: Degustação de PANC nas feiras livres da região metropolitana de Maceió. A - Aplicação de questionários com frequentadores de feiras livres. B - Bolinho de taioba apresentado durante a degustação.

\section{MINICURSOS DE CAPACITAÇÃO}

Em uma nova etapa do projeto de pesquisa, produzimos um formulário online compartilhado a partir das redes sociais do Laboratório de Ecologia, conservação e Evolução Biocultural (LECEB), que foi respondido por 140 pessoas que residem no Estado. O formulário visou entender as principais barreiras ao consumo de PANC e o perfil das pessoas que estariam mais propensas a incluí-las na sua dieta. Entre os nossos resultados mais relevantes, identificamos que a dificuldade em ter acesso às PANC foi a principal barreira ao consumo (mencionado por $42,3 \%$ dos entrevistados). Neste sentido, para tentar atuar no contorno a esta barreira, selecionamos três grupos para cursos de curta duração.

O primeiro grupo foi o de agricultores assentados da reforma agrária, cuja participação em feiras orgânicas e agroecológicas em Maceió é ampla. O grupo foi proveniente de seis municípios da Zona da Mata leste alagoana. O minicurso foi realizado no Assentamento Dom Hélder (Figura 2A), com o intuito de fornecer informações que estimulassem os agricultores/as a consumir e comercializar PANC. Ele ocorreu em 14 de junho de 2018 em parceria com a Rede Mutum de Agroecologia. A rede envolve agentes de diferentes entidades, como Universidades, INCRA, ONGs e movimentos sociais populares, em prol da promoção à produção e comercialização agroecológicas no Estado de Alagoas.

Vinculado ao projeto PANC nas Alagoas ${ }^{1}$, o minicurso contou com cerca de 40 participantes e teve duração de oito horas. Ele foi ministrado pelo Prof. Dr. Valdely Kinupp e se deu em duas etapas: uma apresentação inicial sobre PANC e uma caminhada a quintais das propriedades dos assentados, de maneira a identificar as PANC locais, prover informações sobre elas e a sugerir formas de preparo.

\footnotetext{
O "Projeto PANC nas Alagoas" - já com duas edições, em 2018 e 2019 - foi idealizado pelo Engenheiro Agrônomo Leandro Benatto, a ecochef Sandra Calaça e a artista plástica Synara Holanda, que teve como propósito promover um ambiente institucional de valorização das PANC em Alagoas, contando com parcerias como o IFAL - Murici, o LECEB, o Restaurante Divina Gula e a ABRASEL (na pessoa do André Generoso), a SEMARH (Secretaria do Meio Ambiente e de Recursos Hídricos, na pessoa da Domênica Didier), a EMBRAPA. Estruturado para envolver diferentes segmentos da cadeia agroalimentar, o projeto já tratou desde a identificação das PANC até o mercado e beneficiamento desses recursos.
} 
O segundo grupo foi de estudantes das ciências agrárias, considerando que o exercício de sua profissão poderia levar a uma disseminação futura de informações que estimulem agricultores e extrativistas a trabalhar com PANC. Ele foi ofertado durante o $62^{\circ}$ Congresso Nacional dos Estudantes de Agronomia (CONEA) e XXIV Congresso Latino americano e Caribenho das Entidades Estudantis de Agronomia (CLACEEA), em Setembro de 2019, no Centro de Ciências Agrárias (CECA/UFAL). Com o tema "PANCs are not dead", a equipe de estudantes do LECEB tratou de questões mais gerais a respeito de como um vegetal pode ser considerado PANC ou não, e de todo o caminho percorrido até o acrônimo "PANC" ter sido criado, encerrando o espaço com a degustação às cegas de algumas PANC da região.

O terceiro (Figura 2B) grupo foi formado por estudantes camponeses discentes do Bacharelado em Agroecologia da UFAL no âmbito do PRONERA (Programa nacional de Educação na Reforma Agrária). Os estudantes são todos de assentamentos da reforma agrária ou comunidades quilombolas e, desse modo, sua formação tem como um dos objetivos a disseminação de práticas agroecológicas em suas comunidades. Portanto, a capacitação deste grupo tem o potencial para ampliação da oferta de PANC em feiras do Estado.

Nesse espaço, a equipe do LECEB expôs as metodologias e os resultados dos seus estudos. Após a exposição, foram realizados exercícios para a identificação de PANC com potencial para popularização, de forma que os estudantes foram divididos em grupos de acordo com a região onde moram (Zona da Mata, Agreste ou Sertão). Também fomentamos discussões sobre as principais barreiras para que os agricultores e extrativistas comercializem PANC. Os discentes levantaram aspectos interessantes, entre eles as incertezas sobre a demanda, o que requer o beneficiamento de produtos a base de PANC para conferir maior durabilidade e evitar desperdícios em dias ou semanas nos quais as plantas não forem demandadas.

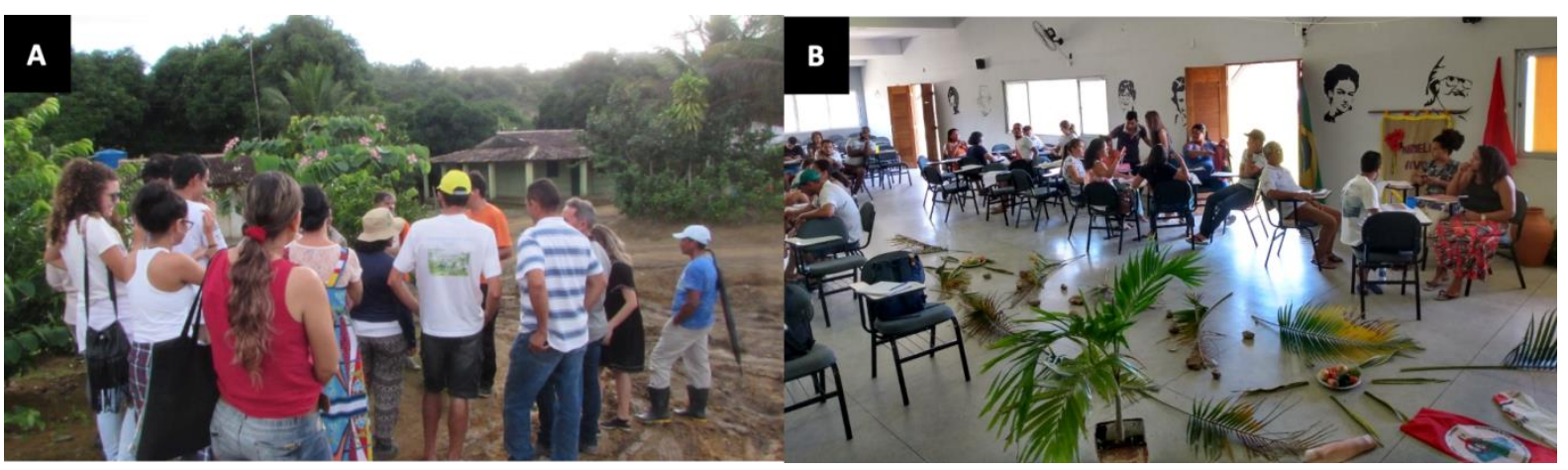

Figura 2: Minicursos organizados pelo LECEB/UFAL. A - Curso para agricultores do Assentamento Dom Helder Câmara (Murici-AL) e assentamentos do entorno. B - Curso para estudantes de Agroecologia (Bacharelado) da UFAL/PRONERA.

\section{POPULARIZAÇÃO DAS PANC: CONCURSO DE CULINÁRIA}

A pesquisa por meio de formulário online apontou que a falta de conhecimento sobre as PANC identificação, formas de preparo etc. - é a segunda maior barreira ao consumo (citada por $29,6 \%$ dos entrevistados), e que o conhecimento prévio sobre este grupo de espécies é principal preditor da disposição a consumir PANC. Para que mais pessoas tivessem acesso ao conceito de PANC e a formas de preparo, promovemos o primeiro concurso de culinária "PANC na Pança" (Figura 3). O concurso foi aberto a pessoas interessadas em culinária com PANC, tendo ou não formação formal em gastronomia.

O concurso foi divulgado nas redes sociais do laboratório, no website da UFAL e na TV aberta de maior audiência no Estado, o que resultou em uma expressiva repercussão. Contamos com seis jurados, entre os quais estavam importantes nomes da gastronomia, nutrição e literatura de Alagoas.

As inscrições foram realizadas via internet e cada candidato deveria enviar uma receita original de prato principal e uma de sobremesa. Assim, a Comissão Julgadora selecionou, com base nas receitas enviadas, seis finalistas para a etapa presencial. A etapa final do concurso aconteceu no restaurante $\mathrm{K}$ Fresh Food em Maceió, no dia 27 de janeiro de 2020 às 19h e contou com cinco dos seis finalistas.

Cada candidato apresentou dois pratos ao júri - um prato principal e uma sobremesa - que foram avaliados separadamente. Primeiro foi avaliado o prato principal de todos os candidatos e em seguida os pratos de sobremesa. 
Após a degustação dos pratos apresentados por todos os participantes, os avaliadores atribuíram suas notas conforme os critérios de avaliação baseados na apresentação, sabor e grau de participação de PANC na receita. A ordem dos finalistas se deu pela soma das notas do prato principal e sobremesa. Os três premiados apresentaram as seguintes receitas: $1^{\circ}$ lugar (Cristiana Purcell) - Tartar com Carpaccio Selado e Panna Cotta de Clitória Com Coulis de Flores Vermelhas; $2^{\circ}$ lugar (Maria Rita Rosa) - Moqueca de Taioba com Banana da Terra e Sorvete de Tapioca com Geleia de Murici; e $3^{\circ}$ lugar (Raquel Maria da Silva) - Escondidinho de Cará com Carne de Jaca Verde e Bolo de Jenipapo com Geleia. Os vencedores receberam premiação em dinheiro e certificados da premiação.

O evento foi transmitido ao vivo para o público nas redes sociais do LECEB (Facebook/Instagram). A mesma TV local que divulgou as inscrições fez a cobertura do evento, que foi transmitida no noticiário matinal e em programa voltado ao meio rural. Também houve menção do evento em portais da mídia local e de instituições alagoanas.

A equipe do laboratório converteu os pratos produzidos na competição em um e-book de receitas, reunindo-as com os ingredientes necessários e o modo de preparo. O livro é mais uma ferramenta de propagação das PANC e dos seus variados usos (Figura 4).

\section{POPULARIZAÇÃO DAS PANC: PRODUÇÃO AUDIOVISUAL}

Ainda no intuito de contribuir para reduzir as barreiras advindas da falta de conhecimento, outra ferramenta utilizada pelo LECEB foi a produção de vídeos educativos. No total, foram quatro vídeos produzidos e seus conteúdos versaram tanto sobre as características - botânicas, nutricionais e gastronômicas - das quatro PANC mencionadas anteriormente, como também sobre as PANC em geral. Os vídeos foram publicados no YouTube (Figura 5) e compartilhados nas diversas redes sociais do LECEB.

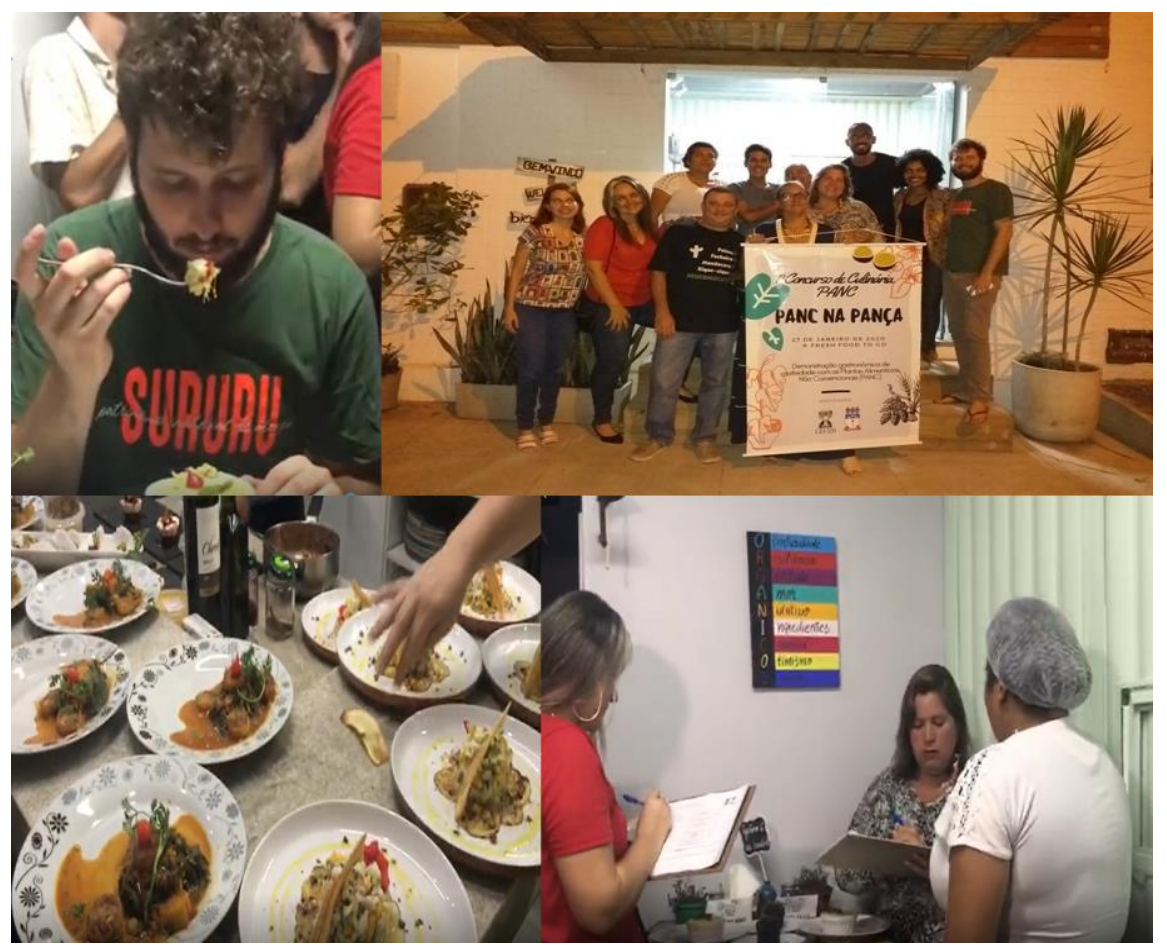

Figura 3: Primeiro Concurso com Plantas Alimentícias Não convencionais "PANC NA PANÇA" - Avaliação dos pratos e equipe de jurados com os participantes da competição. 

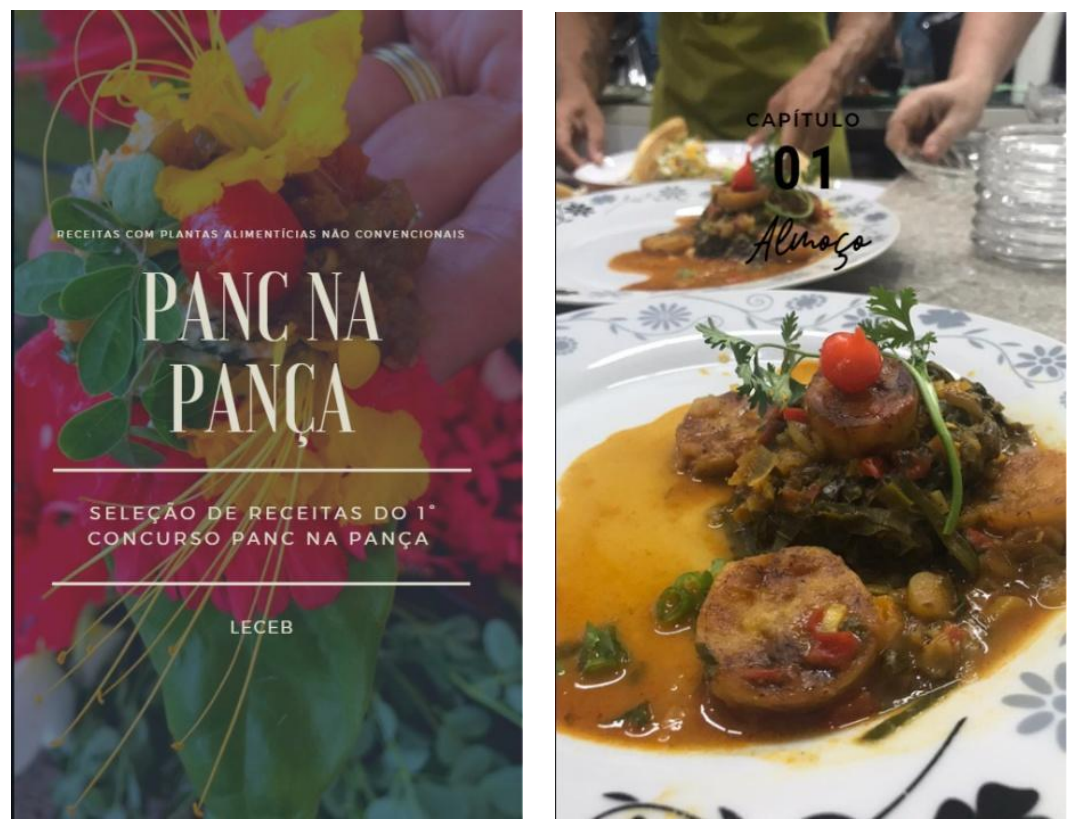

Figura 4: Capa e capítulo 1 do e-book do Primeiro Concurso de Culinária do LECEB, PANC na Pança.

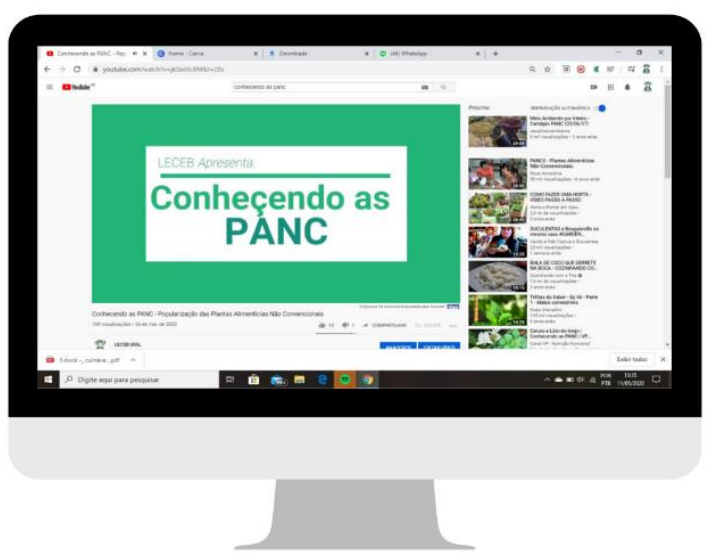

Figura 5: Um dos vídeos do canal do LECEB publicados no YouTube.

\section{CONSIDERAÇÕES FINAIS}

A popularização de PANC em Alagoas é um desafio complexo e requer esforços de múltiplos atores. Assim, o projeto apresentado se soma a excelentes iniciativas no Estado anteriores à nossa. Destacamos as ações da supracitada Rede Mutum no desenvolvimento de cursos e assistência a agricultores e extrativistas, além dos projetos de base agroecológica no âmbito do Instituto Terraviva. Ações de alto alcance e relevância também têm sido promovidas pela Secretaria do Estado do Meio Ambiente e Recursos Hídricos de Alagoas, com projetos coordenados por Domênica Didier, que tem promovido oficinas com feirantes e levado regularmente PANC para degustação em uma das principais feiras agroecológicas de Maceió (Feira da Ponta Verde).

O papel de cooperativas locais também tem sido muito importante para ampliar a demanda por PANC no Estado. Destacamos, neste sentido, a atuação da Associação Aroeira no município de Piaçabuçu, que vêm desenvolvendo capacitações para o beneficiamento e o desenvolvimento de produtos com espécies frutíferas nativas.

As ações do nosso projeto integraram ensino, pesquisa e extensão para contribuir com o cenário de popularização. Desse modo, fomos capazes de alcançar diversos atores das cadeias produtivas de PANC no Estado de Alagoas. Na base da cadeia, trabalhamos com agricultores e extrativistas, que foram familiarizados e incentivados a comercializar estas espécies. No entorno da cadeia, buscamos sensibilizar estudantes, que proverão assistência técnica pautada em um agroextrativismo sustentável. Por fim, no topo da cadeia, realizamos ações que visaram ampliar o conhecimento de potenciais consumidores sobre as PANC. 
Entretanto, algumas ações ainda são necessárias no sentido de ampliar o alcance do material audiovisual produzido, ampliar a oferta de cursos e a diversidade de questões relacionadas às PANC e estabelecer parcerias com outras entidades e instituições do Estado de Alagoas e de outros Estados do Brasil.

\section{AGRADECIMENTOS}

Agradecemos aos moradores do Assentamento Dom Hélder por gentilmente receber o nosso grupo de pesquisa, nos permitindo visitar seus lares e recordações, e aos voluntários que participaram das avaliações sensoriais e entrevistas online. Agradecemos também aos participantes, jurados e facilitadores do $1^{\circ}$ Concurso Culinário "Panc na Pança". Por fim, agradecemos ao CNPq pelo auxílio financeiro ao projeto Bases para o estabelecimento de um programa de popularização das plantas alimentícias não convencionais (442810/2016-4) e pela bolsa de produtividade concedida PMM (302786/2016-3) e a L'Oréal e UNESCO pelas bolsas concedidas a PMM no âmbito do Prêmio Para Mulheres na Ciência do Brasil e International Rising Talents.

\section{REFERÊNCIAS}

BERILLI, S. da S. et al. Avaliação sensorial dos frutos de cultivares de abacaxi para consumo in natura. Revista Brasileira de Fruticultura, Jaboticabal, v. 33, n. especial, p. 592-598, 2011.

CARVALHO, J. M. de et al. Bebida mista com propriedade estimulante à base de água de coco e suco de caju clarificado. Ciência e Tecnologia de Alimentos, Campinas, v. 25, n. 4, p. 813-818, 2005.

DUARTE, G. da R. Levantamento e caracterização das plantas alimentícias não convencionais do Parque Florestal de Monsanto-Lisboa. 2017. 99 f. Dissertação (Mestrado em Ecologia Humana e Problemas Sociais Contemporâneos)-Faculdade de Ciências Sociais e Humanas, Universidade Nova de Lisboa, Lisboa, 2017.

FAO (Food and Agriculture Organization). The secod Report on the state of the world's plant genetic resources for food and agriculture. Roma: FAO, 2015.

FAO (Food and Agriculture Organization). Voluntary guidelines for the conservation and sustainable use of crop wild relatives and wild food plants. Roma: FAO, 2017.

KINNUP, V. F.; LORENZI, H. Plantas alimentícias não convencionais (PANC) no Brasil: guia de identificação, aspectos nutricionais e receitas ilustradas. 1. ed. São Paulo: Instituto Plantarum de Estudos da Flora, 2014.

LAWLESS, H. T.; HEYMANN, H. Sensory evaluation of food: principles and practices. 2. ed. New York: Springer Science \& Business Media, 2010.

MAGDOFF, F.; VAN ES, H. Building soils for better crops: sustainable soil management. 3 ed. The Sustainable Agriculture Research and Education (SARE) Program, with funding from the National Institute of Food and Agriculture. US Department of Agriculture, 2009.

MOREIRA, J. N.; CORREIA, R. C. ; BRANDÃO, W. N. Cultivo da palma no semiárido. Instruções Técnicas da Embrapa Semiárido. EMPBRAPA, 2016.

RANIERI, G. R. et al. Guia prático de PANC - plantas alimentícias não convencionais. 1. ed. São Paulo: Instituto Kairós, 2017.

RAPOPORT, E. et al. Flora y vegetación de una localidad del sur de Mendoza, Argentina. Resultados de un estudio de impacto ambiental por actividades mineras. Multequina, Mendoza, v. 10, n. 1, p. 51-66, 2001.

SOARES, W. L.; PORTO, M. F. S. Estimating the social cost of pesticide use: an assessment from acute poisoning in Brazil. Ecological Economics, v. 68, n. 10, p. 2721-2728, 2009.

VASCONCELOS, A. C. et al. Processamento e aceitabilidade de pães de forma a partir de ingredientes funcionais: farinha de soja e fibra alimentar. Alimentação e Nutrição, Araraquara, v. 17, n. 1, p. 43-49, 2006. 